\title{
The Risk Factors of Polymicrobial Periprosthetic Joint Infection: A Single-Center Retrospective Cohort Study.
}

Hao Li

Chinese PLA General Hospital

Jun Fu

Chinese PLA General Hospital

Wei Chai

Chinese PLA General Hospital

LiBo Hao

Chinese PLA General Hospital

Jiying Chen ( $\nabla$ chenjiyingpla@sina.com )

Chinese PLA General Hospital

ErLong Niu

Chinese PLA General Hospital

Chi Xu

Chinese PLA General Hospital

\section{Research Article}

Keywords: periprosthetic joint infection, total joint arthroplasty, polymicrobial PJI, risk factors

Posted Date: April 16th, 2021

DOl: https://doi.org/10.21203/rs.3.rs-412043/v1

License: (9) (i) This work is licensed under a Creative Commons Attribution 4.0 International License.

Read Full License 
Title: The Risk Factors of Polymicrobial Periprosthetic Joint Infection: A Singlecenter Retrospective Cohort Study.

Running title: The Risk Factors of Polymicrobial Periprosthetic Joint Infection Hao $\mathrm{Li}^{1,2}$, Jun $\mathrm{Fu}^{2}$, Erlong $\mathrm{Niu}^{2}$, Wei Chai ${ }^{2}, \mathrm{Chi}, \mathrm{Xu}^{2}, \mathrm{LiBo} \mathrm{Hao}^{2}$, Jiying Chen ${ }^{2}$

\section{Affiliations:}

1. Medical School of Chinese PLA, Beijing, People's Republic of China;

2. Department of Orthopedic Surgery, The First Medical Center, Chinese PLA General Hospital, Beijing, People's Republic of China;

Key words: periprosthetic joint infection, total joint arthroplasty, polymicrobial PJI, risk factors

Correspondence: Department of Orthopedic Surgery, The First Medical Center, Chinese PLA General Hospital, 28 Fuxing Road, People's Republic of China, China.

\section{Contributor information:}

Hao Li, MD E-mail: lihao_969@qq.com ORCID: 0000-0003-4704-3174

Erlong Niu MD E-mail: 2739976862@qq.com ORCID: 0000-0001-9093-8892

Wei,Chai MD E-mail: chaiweiguanjie@ sina.com ORCID: 0000-0001-8781-9745

Fu Jun MD E-mail: fujun301gk@163.com ORCID ０000-0002-8692-6810

Chi,Xu MD E-mail: zhenyunale@163.com ORCID: 0000-0003-4211-0657

\section{Corresponding author:}

LiBo Hao MD E-mail: haolibo301@sina.comＯRCID: 0000-0003-3052-8714 Jiying, Chen MD E-mail: chenjiyingpla@ sina.com ORCID: 0000-0001-8166-4658 Study design: Hao Li. Data collection/validation: Hao Li and Erlong Niu. Data analysis: Hao Li. Result interpretation: Hao Li. Reporting and editing: Jun Fu and Libo Hao. Final approval of the version to be submitted: Jiying Chen and Libo Hao. 
24 Project guarantor: Jiying Chen and LiBo Hao. The author(s) read and approved the 25 final manuscript.

\section{Conflict of Interest Statement:}

27 Each author certifies that he or she has no commercial associations

28 Ethical review committee statement:

29 This study was performed in accordance with the ethical standards in the 1964

30 Declaration of Helsinki. All methods were carried out in accordance with relevant

31 guidelines and regulations. And Institutional review board approval was obtained

32 prior to the commencement of this study

33

34 
Abstract:

Background: Periprosthetic joint infection is a serious complication after total joint arthroplasty and polymicrobial PJI which compose a subtype of PJI often indicate worse outcomes compared to monomicrobial periprosthetic joint infection. However, a literature review suggested that there were limited number studies evaluating the risk factors of polymicrobial PJI.

Materials and methods: Between 2015 January and 2019 December, a total of 64 polymicrobial PJI patients and 158 monomicrobial PJI patients in a tertiary center were included in this study and corresponding medical records were scrutinized. The diagnosis of PJI was based on 2014 MSIS criteria. Logistic regression was used to identify the association between various variables and polymicrobial PJI and ROC curve was used to identify their efficiency.

Results: The prevalence of polymicrobial PJI is about $28.28 \%$ in our cohorts. After adjusting, the presence of previous revisions (OR, 1.93; $\mathrm{p}<0.01)$, isolation of enterococci (OR, 11.36; $p<0.01)$, isolation of Escherichia coli (OR, 6.55; $p<0.01)$, infection with atypical organisms (OR, 9.85; $\mathrm{p}<0.01)$, infection with gram-negative organisms (OR, 6.33; $p<0.01)$, isolation of streptococcus spp (OR, 1.93; $p<0.01)$, and infection with CNS (OR, 1.93; $\mathrm{p}<0.01)$ were risk factors of polymicrobial PJI compared to monomicrobial PJI. However, knee infection is a protection factor of polymicrobial PJI with an adjusted $\mathrm{OR}=0.0479(\mathrm{p}=0.023)$. 
Conclusion: This study demonstrated that the prevalence of polymicrobial PJI is about 28.28\% in PJI patients. Moreover, the presence of sinus tract and previous joint revisions were risk factors for identifying different bacterial species in the intraoperative specimens. Therefore, in these PJI cases, it is necessary to examine multiple specimens of both intraoperative tissue and synovial fluid for increasing the detection rate and obtaining resistance information.

\section{Backgrounds:}

Total knee arthroplasty (TKA) and total hip arthroplasty (THA) are successful surgeries during the last century but some severe complications occur after total joint arthroplasty (TJA). Periprosthetic joint infection (PJI) is a serious complication after TJA and lays a huge burden on both orthopedic doctors and PJI patients.[1-3]

Polymicrobial PJI, accounting for $6 \%$ to $37 \%$ of PJI, is a subtype of PJI and some studies revealed that polymicrobial PJI was associated with reduced cure rate compared to monomicrobial PJI[1, 2, 4]. The management of polymicrobial PJI is tough and requires repeated revisions, higher treatment cost, administration of board-range antibiotics and multiple antibiotics to fight against PJI pathogens. Therefore, the treatment of polymicrobial PJI is associated with higher motility, higher costs, and more complications[5-8].

However, a literature review suggested that the studies about polymicrobial PJI were limited because of the relatively low occurrence rate of polymicrobial PJI. Despite a fact that some studies revealed that the presence of sinus and certain cultured 
77 pathogens was associated with a higher risk of polymicrobial PJI, there are still some 78 uncertainties regarding the risk factors of polymicrobial PJI[1, 2, 5]. Moreover, few 79 studies revealed the distribution of polymicrobial PJI pathogens in Asian.

80 To address the problems mentioned above, we performed a single-center 81 retrospective study to determine 1) the distribution of pathogens in polymicrobial PJI 82 patients 2) the risk factors associated with risk factors of polymicrobial PJI 


\section{Material and methods:}

\section{Patients:}

Institutional review board approval was obtained prior to the commencement of this study and informed consents were obtained before revisions. Between 2015 and 2019, a total of 843 revision patients were included consequentially in this study initially. And the inclusion criteria were as follows: 1) PJI patients based on the 2014 MSIS criteria 2) No spacer implantation. Exclusion criteria: Culture-negative PJI patients were excluded from this study. The corresponding medical records were extracted and then, these data were scrutinized manually. After selection, a total of 256 PJI patients were included in this study and the process of selection was shown in figure 1.

Moreover, following information was extracted: the age, sex, body mass index (BMI), involved joint, the type of identified pathogens, previous revisions, the nature of the surgical treatment, the presence of sinus tract, the levels of ESR, CRP. Previous revisions were defined as the patients received surgeries on the same joint after primary total joint arthroplasty.

\section{The diagnosis of polymicrobial PJI}

The PJI diagnosis was based on the 2014 Musculoskeletal Infection Society (MSIS) criteria [9]. The patients with PJI in which at least 2 different organisms were isolated from the culture of synovial fluid or intraoperative tissue were considered to have polymicrobial PJI. A total of 64 PJI patients were considered to have polymicrobial PJI according to this definition. 


\section{Culture and microbiological analysis:}

106

107

According to the standard protocol at our institution, specimens obtained was sent for aerobic, anaerobic, and fungal cultures, with a mean number of 5 samples taken from each PJI patient. After culture, the pathogens isolated were identified and antibiotic sensitivity tests (AST) were performed. A matrix-assisted laser desorption-isolation time-of-flight mass spectrometer (VITEK-MS (BioM'erieux)) was used to identify the pathogens isolated from culture.

\section{Statistically analysis:}

The variables were divided into continuous variables and dichotomous data based on the types of data. A normal distribution test was used to evaluate the distribution of continuous variables. The continuous variables were described as means if the normal distribution was achieved. Otherwise, corresponding medians were calculated. Rand sum test and student t-test were used to detect the difference if the corresponding applicable conditions were met. Dichotomous data were described as frequencies and compared by chi-squared test subsequently. Logistic regression was used to evaluate the relationship between the potential risk factors and polymicrobial PJI. OR and adjusted OR were used to evaluate the relative risk of these potential risk factors. The statistical analysis was performed on SPSS (IBM; version 26.0). $\mathrm{P}<0.05$ indicates statistical significance.

\section{Results:}

\section{Demographic characteristic of PJI patients}


The mean ages were 59 and 64.2 years in the polymicrobial PJI and monomicrobial groups, respectively. The percentages of knees were $37.5 \%$ and $59.5 \%$ in these two groups, respectively. The percentage of males in these two groups was $42.2 \%$ and

\section{The risk factors of polymicrobial PJI}

In the univariate the presence of a sinus tract $(\mathrm{OR}, 3.405)$, the presence of previous revisions $(\mathrm{OR}, 2.512)$, knee infection $(\mathrm{OR}, 0.402)$ isolation of enterococci $(\mathrm{OR}$, 2.669;), infection with atypical organisms (OR, 4.371), infection with gramnegative organisms (OR, 2.593), isolation of streptococcus spp (OR, 5.499), and infection with CNS (OR, 1.773)

Moreover, in the multivariate logistic regression analysis, two patient characteristics were found to be the significant independent risk factors of 
polymicrobial PJI: the presence of sinus $(\mathrm{OR}, 2.565 ; \mathrm{p}<0.001)$ and previous revisions $(\mathrm{OR}, 1.954 ; \mathrm{p}=0.039)$. Besides, the knee infection is a protection factor of polymicrobial PJI compared to the hip infection (OR, 0.479; $\mathrm{p}=0.023)$. No other patient characteristics (including joint, sex and BMI) were identified as risk factors. Besides, in the multivariate logistic regression analysis, the adjusted OR of different pathogens was calculated after adjusting the presence of sinus and previous

\section{Discussion:}

Polymicrobial PJI is a serious complication after total joint arthroplasty and often indicates unfavorable prognosis compared to monomicrobial PJI. However, the studies about the risk factors of polymicrobial PJI are limited. This study evaluated the risk factors of polymicrobial PJI and revealed some findings. 

$10.5 \%$ to $19 \%$ of THA PJIs and $9 \%$ to $12.3 \%$ of TKA PJIs in previous studies[1]. We have observed that the percentage of polymicrobial PJI in culture-positive PJI is about $28.82 \%$ and it is consistent with previous reports[10].

The distribution of pathogens in polymicrobial PJI is like that in monomicrobial PJI patients. Staphylococcus spp, including staphylococcus aureus and CNS, is still the most comm isolated pathogen in polymicrobial PJI. However, The presence of sinus tract is associated with higher risk of polymicrobial PJI and this result is consistent with previous studies $[1,2]$. The sinus tract can become a shelter of pathogens and a pathway for causative agents to enter the joint cavity so by which antibiotic can be administrated more precisely.

Some studies suggested that repeated revision was a risk factor of PJI but the association between previous revisions wasn't revealed by other studies $[2,3]$. Here, our studies revealed that the presence of previous revisions was also a risk factor of polymicrobial periprosthetic joint infection. Previous repeated procedures in the same joint can impair the surrounding blood supplies around the joints 
potentially and then, the joints became vulnerable to various pathogens because of the poor status of the soft tissues in these patients. It can be the reason of higher risk of polymicrobial PJI in PJI patients with repeated revisions. Hence, Interestingly, Robert Cantey et. reported that age 65 years or older was associated with polymicrobial PJIs. However, our result suggested that the mean and median ages 65 years or older is 0.491 in the bivariate logistic regression ( $\mathrm{P}$ value $=0.018$ ). To further clarify this association, the confounding variate(the presence of sinus) was adjusted, and the adjusted OR: $0.532(\mathrm{p}=0.044,95 \% \mathrm{CI}:(0.288,0.983))$. After adjusting confounding variate (the presence of sinus and previous revisions), the adjusted $\mathrm{OR}=0.561(\mathrm{p}$ value $=0.068)$. This result suggested that there are potential association between previous revisions and the younger ages. Considering that younger patients require a higher quality of life and they are more aggressive in the choice of surgery than the elder ones, the negative contribution of age to polymicrobial PJIs may be attributed to previous revisions.

To further analyze the relationship between previous revisions and polymicrobial PJI, the reasons of previous revisions were collected and analyzed. Previous PJI is the major cause of previous revisions ( 56 cases), followed by aseptic loosening ( 6 cases). Then, bivariate logistic regression was performed to analysis the relationship between the two factors and polymicrobial PJI. Our result revealed that they were risk factors of 
polymicrobial PJI. However, it is hard to discern whether the revision is the cause or the reason of polymicrobial PJI because several PJI patients received revisions in other joint center where the protocols of pathogens culture is heterogeneous and previous revisions can also be the consequence of polymicrobial PI where polymicrobial PJI are associated with increased number of revisions operations and lower cure rate compared to monomicrobial PJI[7]. However, polymicrobial PJI should be noted when a PJI patient with previous revisions was admitted and then, optimal pathogen detection protocols can be chosen such as repeated joint aspiration and more periprosthetic tissues for culture when revisions are performed[11-13].

In line with prior studies, certain types of pathogens are more likely to be isolated from polymicrobial periprosthetic joint infection compared to monomicrobial PJI such enterococcus, streptococcus, CNS and atypical pathogens[1, 2, 4, 5, 14, 15]. Doctors should take this fact into account to guide antibiotics administration when these pathogens were isolated from PJI patients. Besides, the interesting association between certain pathogens and polymicrobial PJI patients indicated that polymicrobial periprosthetic joint infection can be noted when these pathogens were cultured from PJI patients and board-range antibiotics can be optional in PJI patients with these pathogens. Moreover, we also performed a correlation analysis to explore the positive association between different pathogens in polymicrobial PJI group. Unfortunately, no positive association was detected (not shown in this study). It can be attributed to the relatively small sample size in the PJI group. Therefore, further multi-center studies 
because the clarification on this association can guide empirical antibiotic use more precisely. And molecule diagnostics with high sensitivity such as the metagenomic next-generation sequencing (mNGS) can be used to detect polymicrobial PJI when these pathogens were detected by cultures[16].

There were several limitations in this study. Firstly, this study was performed in a tertiary joint center retrospectively and thus has some inherent limitations and selection bias. For example, previous antibiotics can affect microbiological results but the history of antibiotics administration can't be evaluated comprehensively in this study because of the limited medical records. Therefore, we exclude culture-negative PJI patients from this study in a bid to lower this effect. However, bias was also added after exclusion. Secondly, the prognosis of these PJI patients was not included in this study and this field needs to be explored further. Thirdly, only knees and hips were included in this study and no other joints were evaluated in this study. This nature of the study design can also add some bias to this study and further studies including other joints are necessary. Finally, no co-infective bacterial and fungal PJIs were included in this study but Stevenson et. reported that these patients suffered from unfortunate outcomes. Therefore, the risk factors of co-infective bacterial and fungal PJIs need to be explored further[17].

\section{Conclusions:}

Polymicrobial PJI, which occurred at a relative low rate, is a serious complication after total joint arthroplasty but the studies about polymicrobial PJI was limited. Here, our study reveals some risk factors of polymicrobial periprosthetic joint indention. In 
line with previous, polymicrobial PJI was associated with the presence of sinus tract.

256

257

258

259

260

261

262

263

264

265

266

267

268

269

270

271

272

273

274

275

Besides, we found that hip PJI and the presence of previous revisions were also risk

factors of polymicrobial PJI. Moreover, certain types of pathogens such as CNS, enterococcus, streptococcus spp and atypical pathogens were more common in the polymicrobial PJI than those in the monomicrobial PJI patients. These risk factors should be considered when antibiotics administration was performed.

\section{Acknowledgments:}

The authors would like to thank Tao Deng for his participation in the data collection process of this study.

\section{Funding:}

National Key Research and Development Program of China (No.2020YFC2004900)

\section{Availability of data and materials:}

All data and materials were in full compliance with the journal's policy. And the data were obtained in Department of Orthopedic Surgery, The First Medical Center, Chinese PLA General Hospital. The datasets used and during the current study are available from the corresponding author on reasonable request.

\section{Abbreviations:}

TJA: Total joint arthroplasty

PJI: periprosthetic joint infection 
MSIS: Musculoskeletal Infection Society criteria

CNS: coagulase negative staphylococci

\section{Authors' information:}

Hao Li, Rui LI, LL Li, Wei Chai, Chi Xu, LiBao Hao, and Jiying Chen

come from the Department of Orthopedics, Chinese People's Liberation Army General Hospital (301 Hospital), 28 Fuxing Rd., 100853, Beijing, PR China.

\section{Ethics approval and consent to participate}

This study was approved by the institutional review board of our hospital

(Chinese People's Liberation Army General Hospital). Institutional review

board approval was obtained prior to the commencement of this study and

287 informed consents were obtained before revisions.

\section{Consent for publication}

289 We have obtained consent to publish from the participants.

\section{Competing interests}

291 All authors declare that they have no competing interests.

\section{Authors' Contributions :}

Study design: Hao Li. Data collection/validation: Hao Li and Erlong Niu.

Data analysis: Hao Li. Result interpretation: Hao Li. Reporting and editing: 
295 Jun Fu and Libo Hao. Final approval of the version to be submitted: Jiying 296 Chen and Libo Hao. Project guarantor: Jiying Chen and LiBo Hao. The 297 author(s) read and approved the final manuscript.

299 Contributor information:

300 Hao Li, MD E-mail: lihao_969@qq.com ORCID: 0000-0003-4704-3174

301 Erlong Niu MD E-mail: 2739976862@qq.comＯRCID： 0000-0001-9093-8892

302 Wei,Chai MD E-mail: chaiweiguanjie@ sina.com ORCID: 0000-0001-8781-9745

303 Fu Jun MD E-mail: fujun301gk@163.com ORCID０000-0002-8692-6810

304 Chi,Xu MD E-mail: $\quad$ zhenyunale @ 163.com ORCID: $\quad$ 0000-0003-4211-0657

305 Corresponding author:

306 LiBo Hao MD E-mail: haolibo301@ @sina.com ORCID： 0000-0003-3052-8714

307 Jiying, Chen MD E-mail: chenjiyingpla@ sina.com ORCID: 0000-0001-8166-4658 


\section{Reference:}

1. Tan TL, Kheir MM, Tan DD, Parvizi J. Polymicrobial Periprosthetic Joint

312 Infections: Outcome of Treatment and Identification of Risk Factors. The Journal 313 of bone and joint surgery American volume. 2016;98(24):2082-8.

314 2. Marculescu CE, Cantey JR. Polymicrobial prosthetic joint infections: risk factors 315 and outcome. Clinical orthopaedics and related research. 2008;466(6):1397-404.

316 3. Kapadia BH, Berg RA, Daley JA, Fritz J, Bhave A, Mont MA. Periprosthetic joint 317 infection. Lancet (London, England). 2016;387(10016):386-94.

318 4. Carrega G, Bartolacci V, Burastero G, Casalino Finocchio G, Grappiolo G, 319 Salomone $\mathrm{C}$, et al. Etiology of prosthetic joint infections in a tertiary care centre in 320 Italy. Le infezioni in medicina. 2008;16(4):204-8.

321 5. Bozhkova S, Tikhilov R, Labutin D, Denisov A, Shubnyakov I, Razorenov V, et 322 al. Failure of the first step of two-stage revision due to polymicrobial prosthetic 323 joint infection of the hip. Journal of orthopaedics and traumatology : official 324 journal of the Italian Society of Orthopaedics and Traumatology. 2016;17(4):36932576.

326 6. Kavolus J], Cunningham DJ, Rao SR, Wellman SS, Seyler TM. Polymicrobial 327 Infections in Hip Arthroplasty: Lower Treatment Success Rate, Increased Surgery, 328 and Longer Hospitalization. The Journal of arthroplasty. 2019;34(4):710-6.e3.

329 7. Wimmer MD, Friedrich MJ, Randau TM, Ploeger MM, Schmolders J, Strauss $330 \mathrm{AA}$, et al. Polymicrobial infections reduce the cure rate in prosthetic joint infections: 
outcome analysis with two-stage exchange and follow-up $\geqslant$ two years.

332 International orthopaedics. 2016;40(7):1367-73.

333 8. Peel TN, Cheng AC, Lorenzo YP, Kong DC, Buising KL, Choong PF. Factors

334 influencing the cost of prosthetic joint infection treatment. The Journal of hospital 335 infection. 2013;85(3):213-9.

9. Parvizi J, Zmistowski B, Berbari EF, Bauer TW, Springer BD, Della Valle C], et al. New definition for periprosthetic joint infection: from the Workgroup of the Musculoskeletal Infection Society. Clinical orthopaedics and related research. 2011;469(11):2992-4.

10. Villa JM, Pannu TS, Theeb I, Buttaro MA, Oñativia Jl, Carbo L, et al.

341 International Organism Profile of Periprosthetic Total Hip and Knee Infections. The 342 Journal of arthroplasty. 2021;36(1):274-8.

343 11. Boyle KK, Kapadia M, Chiu YF, Khilnani T, Miller AO, Henry MW, et al. Are

344 Intraoperative Cultures Necessary If the Aspiration Culture Is Positive? A 345 Concordance Study in Periprosthetic Joint Infection. The Journal of arthroplasty. 3462021.

347 12. Imagama T, Nakashima D, Seki K, Seki T, Matsuki Y, Yamazaki K, et al. 348 Comparison of bacterial culture results of preoperative synovial fluid and 349 intraoperative specimens in patients with joint infection. Journal of infection and 350 chemotherapy : official journal of the Japan Society of Chemotherapy. 2020.

351 13. Schulz P, Dlaska CE, Perka C, Trampuz A, Renz N. Preoperative synovial fluid 352 culture poorly predicts the pathogen causing periprosthetic joint infection. 
Infection. 2020.

14. Renz N, Trebse R, Akgün D, Perka C, Trampuz A. Enterococcal periprosthetic

355 joint infection: clinical and microbiological findings from an 8-year retrospective 356 cohort study. BMC infectious diseases. 2019;19(1):1083.

357 15. Pasticci MB, Di Filippo P, Pasqualini L, Mencacci A, Pallotto C, Malincarne L, et 358 al. Tolerability and efficacy of long-term treatment with daptomycin, ceftazidime 359 and colistin in a patient with a polymicrobial, multidrug-resistant prosthetic joint 360 reinfection: a case report. Journal of medical case reports. 2014;8:186.

361 16. Tarabichi M, Shohat N, Goswami K, Alvand A, Silibovsky R, Belden K, et al. 362 Diagnosis of Periprosthetic Joint Infection: The Potential of Next-Generation 363 Sequencing. The Journal of bone and joint surgery American volume. $364 \quad 2018 ; 100(2): 147-54$

365 17. Sidhu MS, Cooper G, Jenkins N, Jeys L, Parry M, Stevenson JD. Prosthetic 366 fungal infections: poor prognosis with bacterial co-infection. The bone \& joint 367 journal. 2019;101-b(5):582-8. 
Figures

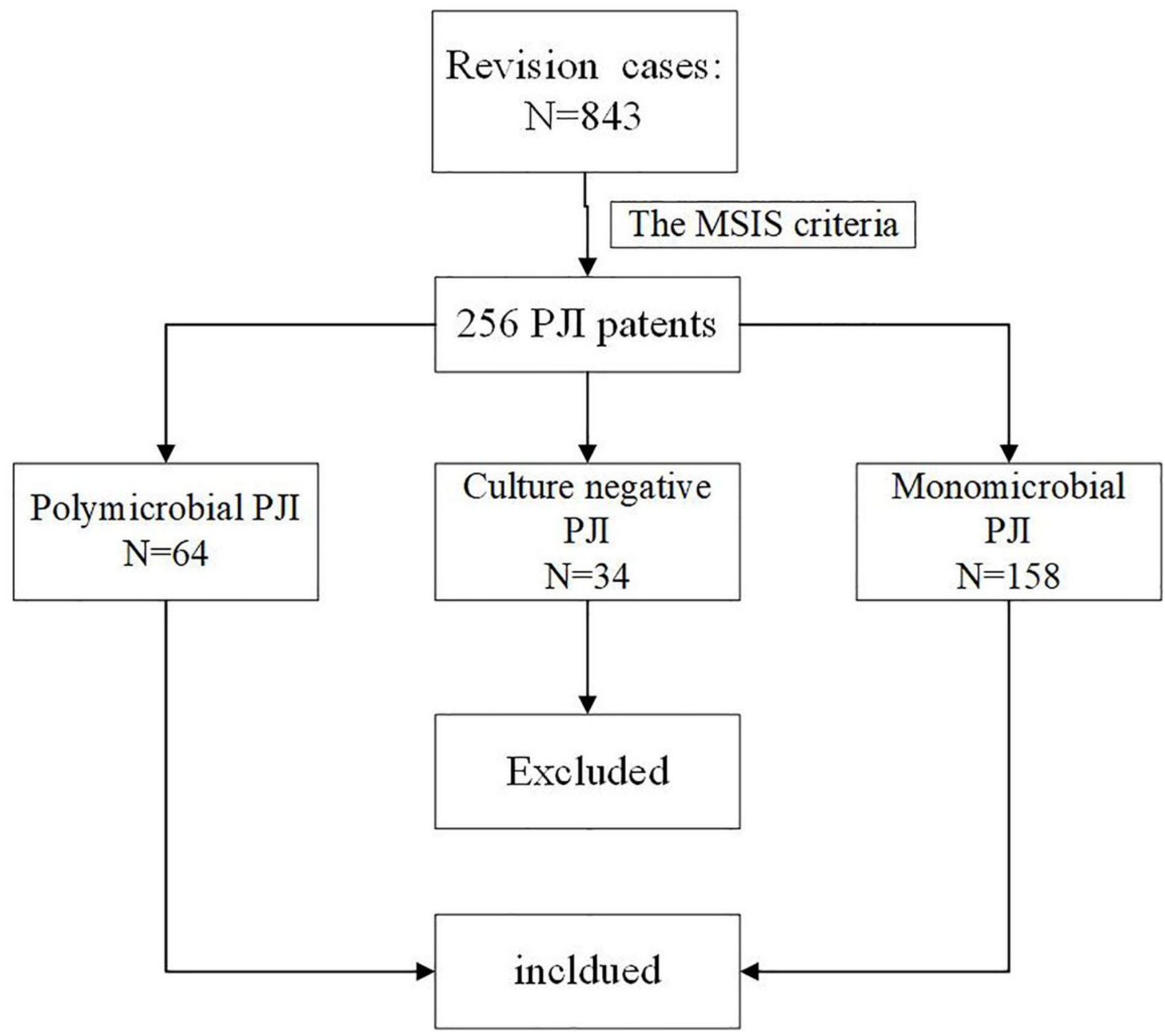

Figure 1

The process of PJI cases included in this study.

\section{Supplementary Files}

This is a list of supplementary files associated with this preprint. Click to download.

- table1.pdf 
- table3.pdf 